\title{
Quisto do Ligamento Amarelo
}

\section{Ligamentum Flavum Cyst}

Joaquim Cruz Teixeira1 ${ }^{1}$ João Levy Melancia²

Autor Correspondente: Joaquim Cruz Teixeira [joaquim.a.teixeira@jmellosaude.pt] Travessa do Castro N³ 3, 1350-070 Lisboa, Portugal

PALAVRAS-CHAVE: Ligamento Amarelo/cirurgia; Ligamento Amarelo/diagnóstico; Quistos/cirurgia; Quistos/diagnóstico; Lombares

KEYWORDS: Cysts/diagnosis; Cysts/surgery; Ligamentum Flavum/diagnosis; Ligamentum Flavum/surgery; Lumbar Vertebrae

Doente do sexo feminino de 72 anos de idade, observada por quadro de dor irradiada ao longo do membro inferior esquerdo no trajeto correspondente aos dermátomos de L5 e S1. A observação neurológica evidenciava abolição do reflexo aquiliano à esquerda sem defeito motor radicular.

Realizou ressonância magnética (RM) lombar em que se documentou uma lesão quística intra-raquidiana, extradural em localização paramediana esquerda e insinuando-se no recesso L5-S1. Apresentava hiperintensidade de sinal nas imagens ponderadas em T2, era isointensa em T1 e com captação marginal de contraste paramagnético, condicionando compressão radicular das raízes de L5 e de S1 homolaterais. Os aspetos imagiológicos descritos são compatíveis com a hipótese diagnóstica de quisto do ligamento amarelo (Fig. 1).

Perante a falência das medidas de terapêutica conservadora para controle da dor radicular e atendendo aos achados imagiológicos acima descritos foi colocada indicação operatória.

Realizou-se fenestração L5-S1 esquerda e flavectomia com remoção de quisto do ligamento amarelo (Fig. 2). No pós-operatório verificou-se resolução sintomatologia dolorosa radicular. Três meses após a cirurgia apresenta-se assintomática e sem evidência de recidiva lesional em RM de controlo.

\footnotetext{
1. Serviço de Neurocirurgia, Hospital CUF Infante Santo, Cluster Tejo, Lisboa, Portugal; Neurocirurgia, Clínica CUF Almada, Almada, Portugal; Neurocirurgia, Clínica CUF Sintra, Sintra, Portugal.

2. Serviço de Neurocirurgia, Hospital CUF Infante Santo, Cluster Tejo, Lisboa, Portugal.

Recebido: 15/02/2019 - Aceite: 14/03/2019
} 

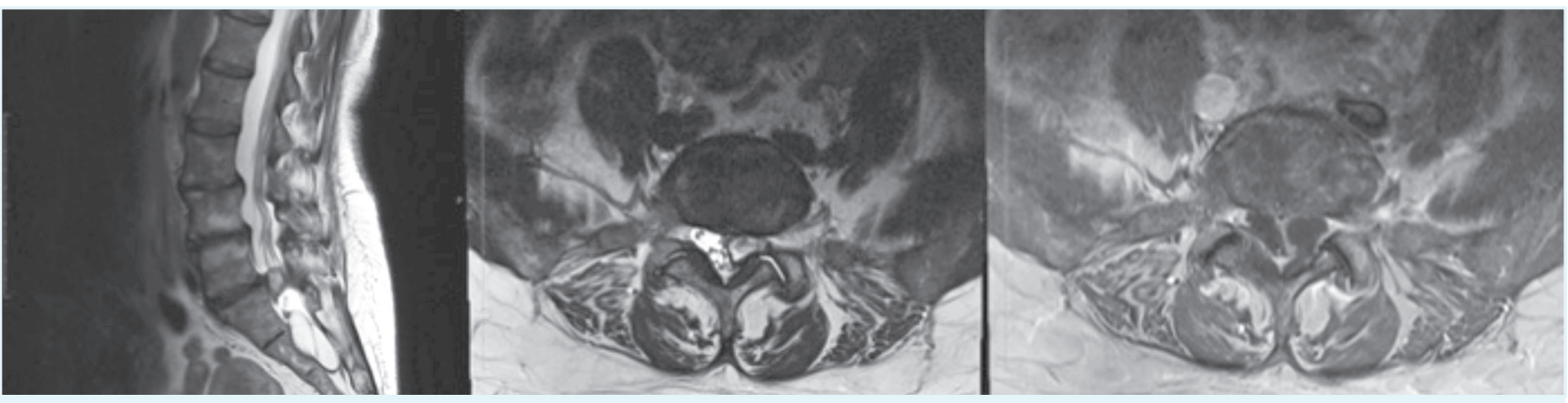

FIGURA 1. Ressonância magnética lombar (Sagital T2/ Axial T2/ Axial T1 com gadolíneo).

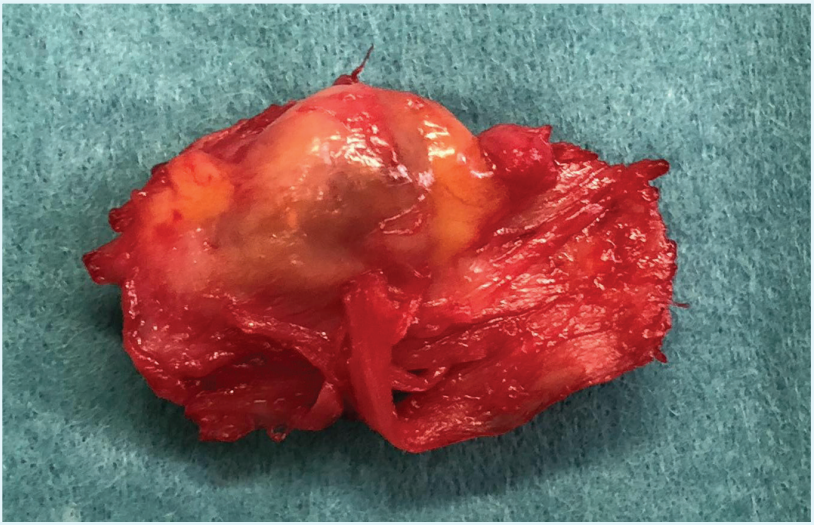

FIGURA 2. Peça operatória.

Os quistos do ligamento amarelo são lesões potencialmente responsáveis por quadros de radiculopatia compressiva lombar, obrigando ao diagnóstico diferencial com o quisto sinovial lombar., ${ }^{1,2}$ Apesar da sua etiologia não ser consensual, parecem resultar de instabilidade segmentar por doença degenerativa discal associada a hipermobilidade facetária, levando a hipertrofia do ligamento amarelo seguida de degenerescência mixoide e consequente formação do referido quisto., ${ }^{1,2}$ A RM é o exame complementar de eleição para o diagnóstico imagiológico dos quistos do ligamento amarelo, que apresentam sinal variável na ponderação T1 e hipersinal em T2. ${ }^{3} \bigcirc$ tratamento conservador apresenta resultados pouco satisfatórios, sendo que o tratamento cirúrgico com excisão total da lesão quística e do ligamento amarelo associado, leva a resolução sintomática e minimiza a hipótese de recorrência. , $^{1,3}$

CONFLITOS DE INTERESSE: Os autores declaram não ter qualquer conflito de interesse na realização do presente trabalho.

FONTES DE FINANCIAMENTO: Não houve qualquer fonte de financiamento na realização do presente trabalho.

CONFIDENCIALIDADE DOS DADOS: Os autores declaram ter seguido os protocolos da sua instituição acerca da publicação dos dados de doentes.
CONFLICTS OF INTEREST: The authors declare that they have no conflicts of interest.

FINANCIAL SUPPORT: This work has not received any contribution, grant or scholarship.

CONFIDENTIALITY OF DATA: The authors declare that they have followed the protocols of their work center on the publication of data from patients.

\section{REFERÊNCIAS}

1. Shah K, Segui D, Gonzalez-Arias S. Midline Ligamentum Flavum Cyst of Lumbar Spine. World Neurosurg. 2018;110:2847. doi: 10.1016/j.wneu.2017.11.075.

2. Cakir E, Kuzeyli K, Usul H, Peksoylu B, Yazar U, Reis A, et al. Ligamentum flavum cyst. J Clin Neurosci. 2004;11:67-9.

3. Taha H, Bareksei Y, Albanna W, Schirmer M. Ligamentum flavum cyst in the lumbar spine: a case report and review of the literature. J Orthop Traumatol. 2010;11:117-22. doi: 10.1007/ s10195-010-0094-y. 